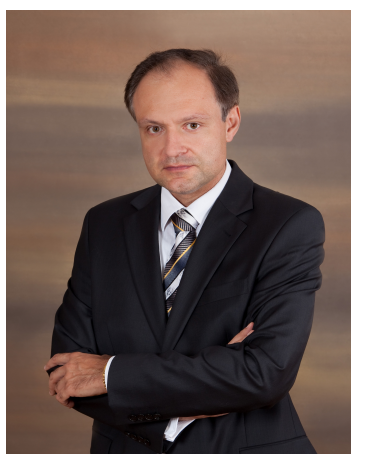

\title{
Can we find active noise control in everyday life soon?
}

Noise is one of the most severe side effects accompanying rapid development of industry and transportation. In industry, noise levels are sometimes so high that they can cause hearing impairment. Even if they are not, they can reduce communication, and hence working efficiency, and may even cause dangerous conditions by masking alert or warning signals. Noisy domestic appliances, like washing machines, dishwashers, refrigerators cause annoyance and affect leisure activities.

There are different passive methods available to protect users against exposure to excessive noise. They include reduction of mechanical impacts, precise fitting of machine parts, use of vibration absorbing mats, isolating noisy mechanisms, isolating the workers, applying acoustical barriers and screens, and finally - personal hearing protection devices. They are not very effective, however, for low-frequency noise.

Such problems are often advanced to justify the use of active noise control. The literature concentrates on developing various solutions employing secondary sound sources distributed in the areas to be protected. The sources are driven by a control system, based on information about the noise in order to reduce it. However, in a diffuse acoustic field, global reduction in an entire enclosure or in a large space around the source is not feasible in practice. Thus, local control in spaces is mostly considered. Such an approach is satisfactory if the system is designed to protect small areas. There are interesting proposals for zones of quiet to move and follow the users or to extend the zones as much as possible. Nevertheless, most companies are interested in offering high acoustical comfort, which definitely is related to global reduction in the entire enclosure or space around the noisy device. So far successful applications of active noise control, although not extensive, are mostly limited to personal hearing protection in headsets or earplugs, ventilation systems, and vehicle cabins.

This editorial intends bring to the attention of the acoustics community, both academic and industrial experts, to take another approach. The main assumptions are to block the noise at the source or not to let it propagate to the protected area and to reduce the noise globally.

One of the successful approaches is the use of active machine casings. The idea is to use the original casing of the device if it is made of thin walls or, otherwise, to enclose the device in an additional thin-wall casing. Then the walls are made to vibrate using structural or dynamic actuators in such a way that the sound propagation is blocked. The approach is an extension of active structural control used for single wall or double-panel walls, developed within project UMO2017/25/B/ST7/02236 funded by the National Science Centre, Poland. However, for the full casing design the overall system is much more challenging. There are usually many walls, which should be controlled at the same time. The walls interact not only through the acoustic field, both inside and outside the casing, but also through structural coupling. Namely, each vibrating wall changes the boundary conditions of the neighbouring walls. One of the crucial problems to make the active casing approach successful is to correctly distribute actuators and sensors to guarantee that all modes are observable and controllable. Laboratory experiments have demonstrated that such methods implemented for real-world devices such as, washing machines can yield over $10 \mathrm{~dB}$ of noise reduction globally. Advances in microelectronics and material engineering should allow for making the cost acceptable.

When working on the active casing method, a methodology to reduce the noise globally without using any controls has been developed. It aims at optimising the location of additional masses and ribs to the casing walls in order to generate deep valleys in the overall casing frequency response in the frequencies, which are dominant in the noise. Although the noise reduction level is smaller than for active control, undoubtedly this solution gives considerable benefits. It follows that this is not really active noise control, but it is definitely a smart noise control solution. This is practically zero-cost, and fully robust, because no sensors, actuators, and electronics are needed, can be thus applied broadly to almost all devices. Even more, shaping the frequency response of the casings is a good way to improve their properties for further active control.

Another solution is active control in openings. The problem concerns different kinds of noise, including transportation and industrial noise. In urban dwellings, as well as in some work places, people feel uncomfortable if windows or doors are closed. Assuming such openings are the dominating paths of noise propagation, an acoustical curtain, similar to a thermal curtain can be created. The research is currently very much advanced and the proposed solutions are gaining the acceptance of residents and urban planners. To be offered for the global market, the efforts concentrate now on totally eliminating sound generators and sensors from the opening. Loudspeakers can be hidden in the edge around the opening. In turn, microphones can be substituted by virtual microphones, which means that the sound pressure at given locations is estimated based on the sound pressure or structural measurement at other locations, e.g. at the edges.

After many years of considerable research activities, it is very encouraging that smart noise reduction solutions have finally convinced people that it is the right time to make them more widely available to the public.

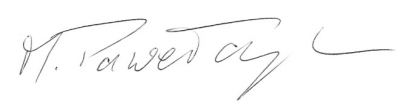

Marek Pawelczyk

IIAV, Vice President for Professional Relations IJAV, Managing Editor 